\title{
Impactos da maloclusão na qualidade de vida de crianças e adolescentes: Uma revisão integrativa
}

\author{
Impacts of malocclusion on the quality of life of children and adolescents: An integrative review \\ Impactos de la maloclusión en la calidad de vida de niños y adolescentes: Una revisión integradora
}

Recebido: 07/06/2021 | Revisado: 15/06/2021 | Aceito: 20/06/2021 | Publicado: 04/07/2021

Savana Ranyella Correia da Silva ORCID: https://orcid.org/0000-0002-2486-8408 Universidade Tiradentes de Alagoas, Brasil E-mail: savana1998@hotmail.com

Vanessa Silva de Moura

ORCID: https://orcid.org/0000-0002-3000-6230 Universidade Tiradentes de Alagoas, Brasil

E-mail: vanessamoura37@gmail.com

Laryssa Kelly Barbosa Ferro Oliveira

ORCID: https://orcid.org/0000-0001-6967-6461 Universidade Tiradentes de Alagoas, Brasil E-mail: laryssakellyferro@gmail.com

Anne Myrelle da Cruz Andrade

ORCID: https://orcid.org/0000-0003-2637-7271

Universidade Tiradentes de Alagoas, Brasil E-mail: annemyrelle@hotmail.com

Luana Ranna Silva Santos

ORCID: https://orcid.org/0000-0002-2361-633X

Universidade Tiradentes de Alagoas, Brasil E-mail: luanaranna2014@gmail.com

Jhenyffer Magarida de Lima Silva

ORCID: https://orcid.org/0000-0002-8192-9377

Universidade Tiradentes de Alagoas, Brasil

E-mail: jhenyffer.margarida@hotmail.com

Anselmo Souza Rastelli

ORCID: https://orcid.org/0000-0002-7095-8869

Universidade Tiradentes de Alagoas, Brasil

E-mail: arastelli92@gmail.com

José Eduardo Melo dos Santos Passos

ORCID: https://orcid.org/0000-0003-4032-2357

Universidade Tiradentes de Alagoas, Brasil E-mail: duduw_9000@hotmail.com

Dayse Andrade Romão

ORCID: https://orcid.org/0000-0002-7884-1657

Universidade Tiradentes de Alagoas, Brasil E-mail: dayseromao@gmail.com

\begin{abstract}
Resumo
As más oclusões possuem etiologia multifatorial, e são caracterizadas pela presença de irregularidades no alinhamento dentário e por desarmonias esqueléticas, que acabam comprometendo a estética do sorriso dos indivíduos, assim como, a qualidade de vida, especialmente nos aspectos psicossociais e nas limitações funcionais. A revisão teve por objetivo proporcionar a síntese de conhecimento sobre a prevalência e as consequências da maloclusão na qualidade de vida de crianças e adolescentes, analisando a literatura no que se refere a casos que venham mostrar a problemática da maloclusão e a intervenção do cirurgião-dentista, visando uma melhor qualidade de vida ao paciente. O presente artigo trata-se de um estudo do tipo revisão integrativa da literatura com caráter qualitativo, onde foi realizado um levantamento bibliográfico nas bases LILACS, SciELO e PubMed, para pesquisa dos artigos foram utilizados os descritores "Má oclusão", "Qualidade de vida", "Articulação temporomandibular", "Crianças" e "Adolescente". Os critérios de inclusão para a seleção da amostra foram artigos publicados em português e inglês, disponíveis na íntegra, entre 2010 e 2020, que retratassem a temática em estudo. Foram identificados cinquenta e um artigos e após a leitura foram selecionados dezessete que atenderam a temática do estudo. A análise da literatura aponta que além de uma possível alteração no desenvolvimento craniofacial, há uma associação da má oclusão aos fatores socioeconômicos, demográficos e cotidianos aos quais interferem em funções biológicas do indivíduo. Nesse sentido, a pesquisa busca apontar a harmonia entre a saúde bucal e o bem-estar do paciente.
\end{abstract}

Palavras-chave: Má oclusão; Qualidade de vida; Articulação temporomandibular; Crianças; Adolescente. 


\begin{abstract}
Malocclusions have a multifactorial etiology, and are characterized by the presence of irregularities in dental alignment and skeletal disharmonies, which end up compromising the aesthetics of the individuals' smiles, as well as the quality of life, especially in terms of psychosocial aspects and functional limitations. The review aimed to provide a synthesis of knowledge about the prevalence and consequences of malocclusion on the quality of life of children and adolescents, analyzing the literature regarding cases that show the problem of malocclusion and the intervention of the dental surgeon, aiming at a better quality of life for the patient. The present article is an integrative literature review type study with a qualitative character, where a bibliographic survey was carried out in the LILACS, SciELO and PubMed databases, to search the articles, the descriptors "Malocclusion", "Quality of life", "Temporomandibular joint", "Children" and "Adolescent". The inclusion criteria for sample selection were articles published in Portuguese and English, available in full, between 2010 and 2020, which portrayed the theme under study. Fifty-one articles were identified and, after reading, seventeen were selected that met the study theme. The analysis of the literature points out that, in addition to a possible change in craniofacial development, there is an association between malocclusion and socioeconomic, demographic and everyday factors that interfere with the individual's biological functions. In this sense, the research seeks to point out the harmony between oral health and the patient's well-being.
\end{abstract}

Keywords: Malocclusion; Quality of life; Temporomandibular joint; Children; Adolescent.

\title{
Resumen
}

Las maloclusiones tienen una etiología multifactorial, y se caracterizan por la presencia de irregularidades en la alineación dentaria y desarmonías esqueléticas, que terminan comprometiendo la estética de las sonrisas de los individuos, así como la calidad de vida, especialmente en aspectos psicosociales y limitaciones funcionales. La revisión tuvo como objetivo brindar una síntesis de conocimientos sobre la prevalencia y consecuencias de la maloclusión en la calidad de vida de niños y adolescentes, analizando la literatura sobre casos que evidencian el problema de maloclusión y la intervención del cirujano dentista, con el objetivo de una mejor calidad. de vida para el paciente. Este artículo es un estudio tipo revisión integradora de la literatura con carácter cualitativo, donde se realizó un relevamiento bibliográfico en las bases de datos LILACS, SciELO y PubMed, para la búsqueda de los artículos se utilizaron los descriptores "Maloclusión", "Calidad de vida", "Articulación temporomandibular", "Niños" y "Adolescente". Los criterios de inclusión para la selección de la muestra fueron artículos publicados en portugués e inglés, disponibles en su totalidad, entre 2010 y 2020, que retrataran el tema en estudio. Se identificaron cincuenta y un artículos y, tras su lectura, se seleccionaron diecisiete que cumplían con la temática de estudio. El análisis de la literatura señala que, además de un posible cambio en el desarrollo craneofacial, existe una asociación entre la maloclusión y factores socioeconómicos, demográficos y cotidianos que interfieren en las funciones biológicas del individuo. En este sentido, la investigación busca señalar la armonía entre la salud bucal y el bienestar del paciente.

Palabras clave: Maloclusión; Calidad de Vida; Articulación Temporomandibular; Niño; Adolescente.

\section{Introdução}

A maloclusão é caracterizada como uma anomalia de desenvolvimento que acomete os dentes e/ou arco dentário, podendo ser considerada como um problema de saúde pública. Em casos mais leves gera uma insatisfação estética e nos mais severos podem ocasionar distúrbios funcionais e incapacitações (Rebouças et al., 2017). Além disso, pode comprometer a função mastigatória e provocar dificuldades de socialização para alguns indivíduos, afetando a autoestima e a autoaceitação (Silva \& Padilha, 2018).

A oclusão normal é denominada como relação harmônica dos elementos dentais. Logo, problemas oclusais são consequências da adaptação da região buco-facial a diversos meios etiológicos, incluindo fatores genéticos, ambientais e hábitos deletérios como suç̧ão de dedo e/ou chupeta na fase das dentaduras decídua e mista, que juntos contribuem para o surgimento da maloclusão, assim como fatores socioeconômicos que também podem estar associados. Em repercussão a várias inferências que podem variar da insatisfação da estética, alterações de fala, deglutição, mastigação, disfunções da ATM (articulação temporomandibular) e dor buco-facial, sua prevalência e impacto contribuem negativamente na vida dos pacientes e em seu convívio social (Johanns et al., 2011; Boeck et al., 2013; Martins et al., 2019).

Tendo em vista que a maloclusão pode ser classificada como problema de saúde pública, as sequelas de seus agravos são circunstanciais, isso se justifica por sua etiologia multifatorial, englobando fatores ambientais e genéticos, que juntos contribuem para o desenvolvimento de diversos tipos de maloclusões. Dessa forma, há a necessidade de um diagnóstico precoce, pois quando realizado são alcançados melhores resultados, tendo em vista que o diagnóstico estabelecido 
precocemente é capaz de orientar o cirurgião-dentista ao tratamento ortodôntico preventivo ou interceptativo adequado ao caso, dispondo do potencial de crescimento da criança. No entanto, o tratamento ortodôntico por ter um maior custo e pelo fato do serviço público de saúde no Brasil não oferecer este tratamento, especialmente quando o mesmo é considerado eletivo, a população com nível socioeconômico mais baixo torna-se impossibilitada de receber este procedimento (Gomes e Silva et al., 2016; Silveira et al., 2016; Guimarães et al., 2018).

Assim, deve ser feita a avaliação desse estado de modo multidimensional, tendo em consideração as particularidades biopsicossociais, além de auxiliar e acompanhar na atenção à saúde. As características do físico e os padrões estéticos são deveras significativos para a sociedade, e estes padrões são analisados tanto na adolescência quanto na infância, sendo períodos com características mais acentuadas, considerando a aceitação e a inserção em um grupo social como papel central (Moreira et al., 2015).

O objetivo deste estudo é analisar casos presentes na literatura que mostram a problemática da maloclusão e como o cirurgião-dentista pode intervir diante de tal situação, visando uma melhor qualidade de vida de crianças e adolescentes.

\section{Material e Métodos}

O presente artigo trata-se de um estudo do tipo revisão integrativa da literatura com caráter qualitativo (Pereira et al., 2018), constituída por uma vasta pesquisa, aprovando assim o envolvimento da literatura teórica e empírica, além de estudos experimentais e não experimentais, para que se obtenha um intenso entendimento sobre o tema abordado.

Foi realizada uma revisão integrativa da literatura, baseada nas seis etapas descritas por Mendes et al., (2008): pergunta norteadora da pesquisa, busca na literatura, categorização dos estudos, avaliação dos estudos, interpretação dos resultados e síntese do conhecimento, definida como aquela em que as pesquisas já publicadas são sintetizadas e geram conclusões sobre o tema em estudo. A questão condutora desta pesquisa foi: "Quais os impactos da maloclusão na qualidade de vida de crianças e adolescentes?".

Para a busca dos artigos foram utilizadas as bases de dados: Literatura Latino-Americana e do Caribe em Ciências da Saúde (LILACS), Scientific Electronic Library Online (SciELO) e US National Library of Medicine (PubMed). Para o levantamento dos artigos foram utilizados como descritores: "Má oclusão", "Qualidade de vida", "Articulação temporomandibular", "Crianças" e "Adolescente". O levantamento bibliográfico ocorreu nos meses de agosto e setembro de 2020.

Inicialmente, a busca pelos descritores foi feita individualmente e em seguida foram realizados os cruzamentos utilizando o operador booleano AND. Os critérios de inclusão para a seleção da amostra foram: artigos publicados em português e inglês, disponíveis na íntegra, publicados e indexados nas referidas bases de dados, nos últimos dez anos (2010 a 2020) e que retratassem a temática em estudo. Os critérios de exclusão seguiram de: artigos publicados antes de 2010, em outros idiomas, além do português e inglês, monografias e trabalhos de conclusão de curso.

\section{Resultados}

Foram analisados vinte artigos científicos que atenderam aos critérios de elegibilidade previamente estabelecidos. $\mathrm{Na}$ base de dados internacional PubMed foram selecionados cinquenta artigos relevantes ao levantamento bibliográfico por meio das palavras chaves "Má oclusão", "Qualidade de vida", "Articulação temporomandibular", "Crianças" e "Adolescente". Porém, através das bases de dados nacionais LILACS e SciELO foi observado um menor números de resultados relevantes aos critérios de inclusão adotados. Além disso, é importante mencionar que, em todas as bases de dados utilizadas, a busca com os termos isolados obteve-se maior retorno de artigos do que o uso combinado de dois ou mais termos. Os artigos encontrados 
foram enumerados conforme a ordem cronológica, identificados e apresentados conforme as normas de referência bibliográfica.

O quadro a seguir expõe os artigos selecionados nas bases de dados.

Quadro 1. Artigos incluídos na revisão integrativa.

\begin{tabular}{|c|c|c|c|c|}
\hline Título & Autor & Ano & Delineamento & Desfecho \\
\hline $\begin{array}{l}\text { As más-oclusões e as desordens da } \\
\text { articulação temporomandibular. }\end{array}$ & $\begin{array}{l}\text { Muller, R. A. et } \\
\quad \text { al. }\end{array}$ & 2010 & Estudo comparativo & $\begin{array}{l}\text { Identificação da relação das maloclusões classe II } \\
\text { e III de Angle com as desordens } \\
\text { temporomandibulares. }\end{array}$ \\
\hline $\begin{array}{l}\text { Há relação de hábitos orais } \\
\text { deletérios com a tipologia facial e } \\
\text { a oclusão dentária? }\end{array}$ & $\begin{array}{c}\text { Johanns, C. M. et } \\
\text { al. }\end{array}$ & 2011 & Estudo transversal & $\begin{array}{l}\text { Evidências de combinação entre o hábito de } \\
\text { ranger dentes com a oclusão dentária Classe I de } \\
\text { Angle. }\end{array}$ \\
\hline $\begin{array}{l}\text { Prevalência de má oclusão em } \\
\text { crianças de } 3 \text { a } 6 \text { anos portadoras } \\
\text { de hábito de sucção de dedo e/ou } \\
\text { chupeta. }\end{array}$ & $\begin{array}{l}\text { Boeck, E. M. et } \\
\quad \text { al. }\end{array}$ & 2013 & $\begin{array}{l}\text { Pesquisa prospectiva } \\
\text { transversal } \\
\text { observacional }\end{array}$ & $\begin{array}{l}\text { A má oclusão pode gerar alterações de fala, } \\
\text { postura, mastigação, respiração, deglutição, } \\
\text { disfunção temporomandibular e problemas } \\
\text { estéticos. }\end{array}$ \\
\hline $\begin{array}{l}\text { Prevalência de má oclusão em } \\
\text { escolares de } 7 \text { a } 9 \text { anos de idade do } \\
\text { Polo } 1 \text { da Rede Municipal de } \\
\text { Ensino em João Pessoa-PB. }\end{array}$ & $\begin{array}{l}\text { Sousa J. P. \& } \\
\text { Sousa S. A. }\end{array}$ & 2013 & Estudo transversal & $\begin{array}{l}\text { As maloclusões repercutem em variados } \\
\text { problemas como: funcionais e estéticos. }\end{array}$ \\
\hline 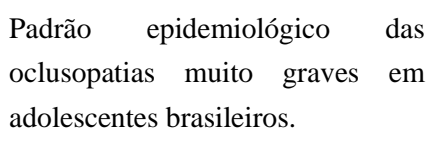 & $\begin{array}{l}\text { Peres, K. G., } \\
\text { Frazão, P. \& } \\
\text { Roncalli, A. G. }\end{array}$ & 2013 & Pesquisa de campo & $\begin{array}{l}\text { Oclusopatias são geradoras de problemas } \\
\text { funcionais, além de gerar alterações estéticas, } \\
\text { psicossociais e de função. }\end{array}$ \\
\hline $\begin{array}{l}\text { Disfunção temporomandibular na } \\
\text { adolescência. }\end{array}$ & Spezzia, S. et al. & 2014 & Revisão de literatura & $\begin{array}{l}\text { A disfunção temporomandibular (DTM) pode ser } \\
\text { causada pela maloclusão, assim com diagnóstico } \\
\text { precoce ajudará a melhorar a qualidade de vida } \\
\text { dos pacientes. }\end{array}$ \\
\hline $\begin{array}{l}\text { Impacto da má oclusão na } \\
\text { dentição decídua e permanente na } \\
\text { qualidade de vida de crianças e } \\
\text { adolescentes: revisão de literatura. }\end{array}$ & $\begin{array}{l}\text { Moreira, A. F. et } \\
\text { al. }\end{array}$ & 2015 & Revisão de literatura & $\begin{array}{l}\text { A má oclusão causa maior impacto na qualidade } \\
\text { de vida quando presente na dentição permanente } \\
\text { do que na decídua, pois nesta faixa etária a } \\
\text { estética ainda não é muito valorizada. }\end{array}$ \\
\hline $\begin{array}{l}\text { Avaliação de sinais e sintomas da } \\
\text { disfunção temporomandibular e } \\
\text { sua relação com a postura cervical. }\end{array}$ & $\begin{array}{l}\text { Viana, M. O. et } \\
\text { al. }\end{array}$ & 2015 & $\begin{array}{l}\text { Estudo transversal e } \\
\text { descritivo com } \\
\text { abordagem quantitativa }\end{array}$ & $\begin{array}{l}\text { Pacientes com DTM apresentam cefaleia, dor } \\
\text { muscular, ruídos na ATM e dificuldade em abrir } \\
\text { a boca, além de existir uma correlação entre este } \\
\text { e a lordose cervical. }\end{array}$ \\
\hline $\begin{array}{l}\text { Gravidade da maloclusão em } \\
\text { adolescentes: estudo de base } \\
\text { populacional no norte de Minas } \\
\text { Gerais, Brasil }\end{array}$ & $\begin{array}{l}\text { Silveira, M. F. et } \\
\text { al. }\end{array}$ & 2016 & $\begin{array}{l}\text { Estudo transversal de } \\
\text { base populacional }\end{array}$ & $\begin{array}{l}\text { As maloclusões consideradas graves são mais } \\
\text { prevalentes em adolescentes com desvantagem } \\
\text { social. Por isso, é de suma importância que } \\
\text { dentre os tratamentos acessíveis para população, } \\
\text { esteja o tratamento ortodôntico, disponibilizado } \\
\text { pelas políticas públicas. }\end{array}$ \\
\hline $\begin{array}{l}\text { Impacto da má oclusão na } \\
\text { qualidade de vida de adolescentes } \\
\text { brasileiros: um estudo de base } \\
\text { populacional. }\end{array}$ & $\begin{array}{l}\text { Gomes e Silva, L. } \\
\text { F. G. et al. }\end{array}$ & 2016 & $\begin{array}{l}\text { Estudo transversal de } \\
\text { base populacional }\end{array}$ & $\begin{array}{l}\text { A má oclusão representada pela necessidade do } \\
\text { tratamento ortodôntico afeta negativamente a } \\
\text { qualidade de vida do adolescente, principalmente } \\
\text { pelo fator estético e condição socioeconômica. }\end{array}$ \\
\hline
\end{tabular}




\begin{tabular}{|c|c|c|c|c|}
\hline $\begin{array}{l}\text { Avaliação objetiva e subjetiva da } \\
\text { necessidade de tratamento } \\
\text { ortodôntico do adolescente e seu } \\
\text { impacto sobre a autoestima. }\end{array}$ & Sharma, A. et al. & 2017 & Estudo transversal & $\begin{array}{l}\mathrm{Na} \text { adolescência, os indivíduos passam por } \\
\text { transformações físicas, psicológicas, emocionais } \\
\text { e de personalidade que os tornam mais } \\
\text { preocupados em relação à estética facial, visto } \\
\text { que a aparência é bastante valorizada nessa } \\
\text { idade. }\end{array}$ \\
\hline $\begin{array}{l}\text { Impacto da cárie dentária, } \\
\text { maloclusão e hábitos orais na } \\
\text { qualidade de vida relacionada à } \\
\text { saúde oral em crianças pré- } \\
\text { escolares. }\end{array}$ & $\begin{array}{c}\text { Carminatti, M. et } \\
\text { al. }\end{array}$ & 2017 & Estudo transversal & $\begin{array}{l}\text { Lesão cariosa, maloclusão e hábitos bucais } \\
\text { deletério causam impactos negativos na } \\
\text { qualidade de vida das crianças, pois prejudicam a } \\
\text { função e a interação social. }\end{array}$ \\
\hline $\begin{array}{l}\text { Fatores individuais associados à } \\
\text { má oclusão em adolescentes. }\end{array}$ & $\begin{array}{l}\text { Rebouças, A. G. } \\
\quad \text { et al. }\end{array}$ & 2017 & $\begin{array}{c}\text { Estudo transversal } \\
\text { analítico quantitativo }\end{array}$ & $\begin{array}{l}\text { O maior índice de cárie e maior chance de } \\
\text { maloclusão severa ou muito severa consistem nos } \\
\text { adolescentes com menor renda familiar e do } \\
\text { grupo étnico preto e pardo. }\end{array}$ \\
\hline $\begin{array}{l}\text { Doenças dentais comuns em } \\
\text { crianças e maloclusão. }\end{array}$ & Zou, J. et al. & 2018 & Revisão de literatura & $\begin{array}{l}\text { Cárie dental, lesões pulpares, lesões periapicais, } \\
\text { traumatismo dentário, alteração no } \\
\text { desenvolvimento e hábitos orais afetam } \\
\text { negativamente na saúde bucal. }\end{array}$ \\
\hline $\begin{array}{l}\text { Impacto da má oclusão na } \\
\text { qualidade de vida relacionada à } \\
\text { saúde bucal em escolares. }\end{array}$ & $\begin{array}{l}\text { Guimarães, S. P. } \\
\text { et al. }\end{array}$ & 2018 & Estudo transversal & $\begin{array}{l}\text { Maloclusão deve ser avaliada na fase de dentição } \\
\text { mista ou permanente inicial, pois o diagnóstico } \\
\text { precoce pode conduzir um tratamento } \\
\text { ortodôntico preventivo sendo favorecido pela } \\
\text { fase de desenvolvimento da criança. }\end{array}$ \\
\hline $\begin{array}{l}\text { A disfunção temporomandibular } \\
\text { afeta a qualidade de vida? }\end{array}$ & Trize, D. M. et al. & 2018 & Estudo comparativo & $\begin{array}{l}\text { Pacientes com disfunção temporomandibular } \\
\text { possuem qualidade de vida afetada } \\
\text { negativamente pela presença de dor e efeitos na } \\
\text { saúde física e mental. }\end{array}$ \\
\hline $\begin{array}{l}\text { Abordagens da produção científica } \\
\text { odontológica brasileira sobre os } \\
\text { efeitos da má oclusão e seus } \\
\text { tratamentos na qualidade de vida. }\end{array}$ & $\begin{array}{l}\text { Silva, A. L. do Ó } \\
\text { \& Padilha, W. W. } \\
\text { N. }\end{array}$ & 2018 & Estudo transversal & $\begin{array}{l}\text { A má oclusão além de afetar a função } \\
\text { mastigatória, causa diversos problemas, como a } \\
\text { socialização, baixa autoestima e aceitação. Por } \\
\text { outro lado, o tratamento adequado resulta em } \\
\text { uma melhora na qualidade de vida dos } \\
\text { indivíduos. }\end{array}$ \\
\hline $\begin{array}{l}\text { Associação entre má oclusão, cárie } \\
\text { e higiene oral em crianças de } 6 \text { a } \\
12 \text { anos residentes no subúrbio da } \\
\text { Nigéria. }\end{array}$ & $\begin{array}{l}\text { Kolawole K. A. \& } \\
\text { Folayan, M. O. }\end{array}$ & 2019 & Estudo transversal & $\begin{array}{l}\text { A má oclusão pode ser associada à cárie e a } \\
\text { gengivite, o que justifica a recomendação do } \\
\text { tratamento ortodôntico para promover a saúde } \\
\text { oral. }\end{array}$ \\
\hline $\begin{array}{l}\text { Má oclusão e vulnerabilidade } \\
\text { social: estudo representativo de } \\
\text { adolescentes de Belo Horizonte, } \\
\text { Brasil. }\end{array}$ & $\begin{array}{l}\text { Martins, L. P. et } \\
\text { al. }\end{array}$ & 2019 & $\begin{array}{l}\text { Estudo transversal } \\
\text { representativo }\end{array}$ & $\begin{array}{l}\text { A má oclusão é um problema de alta prevalência } \\
\text { na saúde pública. Afeta principalmente } \\
\text { indivíduos de classe baixa, além de interferir na } \\
\text { qualidade de vida dos mesmos. }\end{array}$ \\
\hline $\begin{array}{l}\text { Existe uma relação entre má } \\
\text { oclusão e bullying? Uma revisão } \\
\text { sistemática. }\end{array}$ & $\begin{array}{c}\text { Tristão, S. K. P. et } \\
a l\end{array}$ & 2020 & Revisão sistemática & $\begin{array}{l}\text { A maloclusão extrema tem relação com a } \\
\text { ocorrência de bullying entre crianças e } \\
\text { adolescentes. }\end{array}$ \\
\hline
\end{tabular}

Fonte: Autores (2020). 
No quadro um (1) é possível verificar que os artigos foram publicados entre 2010 a 2020, nos quais 20 foram selecionados e organizados por meio de fichamento, em ordem numérica crescente, tendo um (1) artigo de pesquisa de campo, um (1) artigo de pesquisa prospectiva transversal observacional, um (1) artigo de revisão sistemática, um (1) artigo de estudo transversal representativo, um (1) artigo estudo transversal analítico quantitativo, um (1) artigo de estudo transversal e descritivo com abordagem quantitativa, dois (2) artigos de estudo comparativo, dois (2) artigos de estudo transversal de base populacional, três (3) artigos de revisão de literatura e sete (7) artigos de estudo transversal, possibilitando assim a aproximação inicial com o tema. Em seguida, os artigos foram submetidos à leitura na íntegra, tendo como finalidade proporcionar uma melhor busca dos dados apresentados pelos artigos, além de visar uma realização de análise interpretativa.

\section{Discussão}

Entende-se maloclusão como uma desordem do desenvolvimento do complexo craniofacial, determinada como a disposição dos dentes no arco dentário e sua correlação com as bases ósseas e estruturas relacionadas de forma desarmônica. Essa desordem atinge os ossos maxilares, língua, músculos faciais, dentes decíduos e permanentes, causando alterações funcionais como respiração, disfunções temporomandibulares, (Boeck et al., 2013) fala, mastigação, deglutição, dificuldade no desempenho diário de uma simples higienização e insatisfações estéticas, que muitas vezes fazem com que o paciente apresente sequelas psicossociais, prejudicando as relações sociais e desenvolvendo um impacto negativo na qualidade de vida desses indivíduos (Guimarães et al., 2018; Silva \& Padilha, 2018).

Muitas razões podem favorecer para o aparecimento dessa desordem como: aspectos hereditários e ambientais, cárie dentária, lesões pulpares e periapicais, traumatismo dentário, anormalidade do desenvolvimento e hábitos orais; os quais podem ser resultados da interação dos fatores hereditários e ambientais. As maloclusões mais comumente encontradas nas clínicas foram overbite profundo, desvio da linha média, overjet excessivo, mordida cruzada anterior, desalinhamento, espaço e mordida aberta (Zou et al., 2018).

Estes problemas ocupam a terceira posição na escala de prioridades dos problemas orais, proposta pela Organização Mundial da Saúde, com prevalência variando de $20 \%$ a $100 \%$ em concordância com diferentes autores (Peres, Frazão \& Roncalli, 2013; Zou et al., 2018), por isso as maloclusões estão sendo consideradas como um agravo à saúde pública em nível mundial, sendo justificada por sua vasta predominância e por desenvolver consequências negativas na vida dos indivíduos. A OMS (organização mundial da saúde) define a qualidade de vida como "Percepção do indivíduo de sua posição na vida, no contexto da cultura e sistema de valores nos quais ele vive e em relação aos seus objetivos, expectativas, padrões e preocupações" e o conceito de saúde para criança e adolescente como algo que abrange o funcionamento físico, emocional e social da criança e da família (Moreira et al., 2015).

$\mathrm{Na}$ adolescência, os indivíduos passam por transformações físicas, psicológicas, emocionais e de personalidade que os tornam mais preocupados em relação à estética facial, visto que a aparência é bastante valorizada nessa idade. Assim, um sorriso desarmônico ou uma deformidade facial causam impactos na autoestima do adolescente, fazendo com que ele evite sorrir em público, causando problemas de autoconfiança e dificuldade em aceitação, podendo os tornar adultos tímidos em contatos sociais e sem predisposição de interagir em sociedade (Sousa \& Sousa, 2013; Silva \& Padilha, 2018).

O sexo feminino tende a ter um maior impacto na qualidade de vida associada à saúde oral, onde as ações que expõem os elementos dentais tornam-se limitadas pelo constrangimento. Assim, de acordo com estudos, os indivíduos do sexo feminino inclinam-se a terem maiores impactos psicossociais que estão relacionados à maloclusões em comparação ao sexo masculino (Sharma et al., 2017). 
Desse modo, este estado pode afetar a qualidade de vida do paciente, levando em consideração não apenas a saúde física, mas também as implicações no contexto social, pois problemas de autoaceitação e baixa autoestima podem levar o paciente a quadros de ansiedade e depressão (Kolawole \& Folayan, 2019).

A cárie precoce da infância é uma das doenças mais prevalentes, sendo suas consequências uma das causas mais comumente procuradas no atendimento odontológico da criança. No caso de perda precoce do elemento dental pode gerar a migração desse elemento, alterando assim as características da oclusão do indivíduo (Rebouças et al., 2017). Se a lesão cariosa não for tratada pode ocasionar a redução da carga mastigatória ou a mastigação assimétrica, alterando a disposição dos contatos oclusais e mastigação unilateral podendo comprometer o desenvolvimento e crescimento da face, resultando em maloclusões e deformidades dento-faciais (Zou et al., 2018).

Sendo assim, é de extrema importância a avaliação do cirurgião-dentista, principalmente na fase da dentição mista, observando os padrões normais e as desordens nesta faixa etária, para que seja possível manter uma dentição saudável e diagnosticar/tratar precocemente as maloclusões, visto que as alterações oclusais podem persistir na dentição permanente se nenhuma conduta for adotada (Sharma et al., 2017; Zou et al., 2018).

Em relação às crianças, a maloclusão não afeta significativamente a qualidade de vida, principalmente quando se trata dos dentes decíduos. Nessa faixa etária a estética ainda não é muito valorizada e as crianças não se apegam aos dentes temporários, pois sabem que estes cairão e serão substituídos por outros (Trize et al., 2018).

De acordo com a literatura crianças e adolescentes com maloclusão, principalmente as que atingem os dentes anteriores como mordida aberta, sobremordida profunda, sobressalência da maxila e diastemas ou até mesmo dentes ausentes (Moreira et al., 2015; Tristão et al., 2020), tendem a sofrer bullying com maior frequência do que aqueles que apresentam normoclusão. Sendo o bullying um problema com decorrências graves e duradouras, suas vítimas podem apresentar sintomas como ansiedade, solidão e depressão, e sentem receio de ir à escola, local à qual a vítima sente-se desagradável e insegura, podendo afetar seu desempenho escolar (Tristão et al., 2020).

Aspectos socioeconômicos e escolaridade dos indivíduos influenciam diretamente na condição da saúde oral, pois esses fatores estão associados ao grau de conhecimento dos hábitos de vida saudáveis e, consequentemente do reconhecimento em maior ou menor grau da necessidade de assistência odontológica (Rebouças et al., 2017).

A compreensão sobre a distribuição da maloclusão na população e a identificação de fatores e condições a ela associados permite a construção de modelos para entender a sua ocorrência e colaborar na criação de políticas públicas (Rebouças et al., 2017). Além disso, discute-se sobre a oclusão como fator etiológico também da DTM (disfunção temporomandibular), mas não existem estudos na literatura que comprovem que a maloclusão esteja diretamente ligada ao desenvolvimento de disfunções temporomandibulares. Quando a dentição não se encontra em harmonia com a articulação e com os movimentos da mandíbula, podem levar a interferências, que são contatos oclusais indesejáveis, influenciando no aparecimento de DTM (Spezzia et al., 2014).

Pacientes com DTM podem ser estudados através de sintomas e sinais característicos. Os sinais são considerados achados clínicos relacionados com DTM, verificados pela avaliação clínica. Os sintomas são sinais dos quais as pessoas notam, sendo capazes de referi-los. Dentre os sintomas e sinais mais frequentes de DTM observa-se que a cefaleia foi a mais frequente, seguida pela dor muscular e pelos ruídos na ATM (Viana et al., 2015). De acordo com relatos literários a falta de conforto ao mastigar e/ou morder revelou correlação considerável com o grupo de pacientes portadores de DTM. A análise de regressão logística aponta que a dor resultante da DTM está associada ao ranger dos dentes e a outros hábitos parafuncionais (Carminatti et al., 2017).

De acordo com Muller et al., (2010) indivíduos classificados com Classe II de Angle normalmente relatam travamento na articulação temporomandibular (ATM), porém este travamento aparece com maior frequência em indivíduos 
com Classe III de Angle, os quais adicionalmente relatam dor à palpação. Os estudos apontam que o trespasse topo a topo demonstra envolvimento com dores na articulação temporomandibular e dores faciais, além de que os indivíduos com mordida aberta apresentaram maior tendência ao travamento da ATM nos movimentos oclusais, assim como dores no musculo temporal durante a palpação. Ademais, é visto que a mordida cruzada tem relação com a presença de cefaleias, pois produz um estresse e uma hiperatividade dos músculos sendo adjuntos ao surgimento dos sinais e sintomas da disfunção temporomandibular.

\section{Considerações Finais}

Os hábitos parafuncionais são os fatores etiológicos mais comuns para o início das alterações oclusais, tendo em vista outros influenciadores como hábitos bucais e socioeconômicos, além de diversas manifestações patológicas, tornando o problema da maloclusão um dos maiores em saúde bucal do Brasil e trazendo consequências funcionais e psicossociais para os pacientes. Sendo assim, é fundamental que indivíduos com uma menor renda familiar e com maior vulnerabilidade a problemas de saúde bucal possuam acesso ao tratamento ortodôntico permitindo o alcance à equidade em saúde bucal.

Cabe salientar a importância da avaliação do cirurgião-dentista no diagnóstico do paciente, acompanhamento do caso e encaminhamento para tratamento adequado, principalmente nos casos de dentição mista, onde o tratamento precoce evitará que afete a dentição permanente.

Por fim, há necessidade de que trabalhos posteriores tenham um maior aprofundamento quanto ao diagnóstico precoce de crianças e adolescentes viventes em regiões que possuem menor grau de assistência odontológica, visto que, os fatores socioeconômicos estão diretamente ligados à condição de saúde bucal, para que assim seja possível estabelecer um tratamento preventivo ou interceptativo, diminuindo as chances desse público apresentar algumas dessas consequências.

\section{Referências}

Boeck, E. M., Pizzol, K. E., Barbosa, E. G. P., Pires, N. C. A. \& Lunardi, N. (2013). Prevalência de má oclusão em crianças de 3 a 6 anos portadoras de hábito de sucção de dedo e/ou chupeta. Rev. odontol. UNESP. 42(2). 110-116. https://www.scielo.br/j/rounesp/a/JpCsnFz9QBn6PLcKdsy4xDx/abstract/?lang=pt

Carminatti, M., Lavra, B. P., Franzon, R., Rodrigues, J. A., Araújo, F. B. \& Gomes, E. (2017). Impacto da cárie dentária, maloclusão e hábitos orais na qualidade de vida relacionada à saúde oral em crianças pré-escolares. Audiol. Commun. Res. 22:e1801. 1-8. https://doi.org/10.1590/2317-6431-2016-1801

Gomes e Silva, L. F., Thomaz, E. B. A. F., Freitas, H. V., Pereira, A. L. P., Ribeiro, C. C. C. \& Alves, C. M. C. (2016). Impact of Malocclusion on the Quality of Life of Brazilian Adolescents: A Population-Based Study. PLoS ONE. 11 (9): e0162715. 1-13. https://doi.org/10.1371/journal.pone.0162715

Guimarães, S. P. A., Jorge, K. O., Fontes M. J. F., Ramos-Jorge, M. L., Araújo, C. T. P., Ferreira, E. F., Melgaço, C. A., Zarzar, P. M. (2018). Impact of malocclusion on oral health-related quality of life among schoolchildren. Braz. oral. res. 32:e95. 1-9. https://doi.org/10.1590/1807-3107bor-2018.vol32.0095

Johanns, C. M., Silverio, K., Furkim, A. M. \& Marchesan, I. (2011). Há relação de hábitos orais deletérios com a tipologia facial e a oclusão dentária? Rev. CEFAC. 13(6). 1095-1102. https://doi.org/10.1590/S1516-18462011005000060

Kolawole, K. A. \& Folayan, M. O. (2019). Association between malocclusion, caries and oral hygiene in children 6 to 12 years old resident in suburban Nigeria. BMC Oral Health. 19(1). 1-9. https://doi.org/10.1186/s12903-019-0959-2

Martins, L. P., Bittencourt, J. M., Bendo, C. B., Vale, M. P. \& Paiva, S. M. (2019). Má oclusão e vulnerabilidade social: estudo representativo de adolescentes de Belo Horizonte, Brasil. Ciênc. saúde colet. 24(2). 393-400. https://doi.org/10.1590/1413-81232018242.33082016

Mendes, K. D. S., Silveira, R. C. C. P. \& Galvão, C. M. (2008). Revisão Integrativa: método de pesquisa para a incorporação de evidências na saúde e na enfermagem. Texto Contexto Enferm. 17(4). 758-764. https://doi.org/10.1590/S0104-07072008000400018

Moreira, A. F., Pinto, L. S., Pinto, K. V. A., Côrreia, P. G., Jeziorski, S. A. Z., Velasque K. S. \& Rosa, D. P. (2015) Impacto da má oclusão na dentição decídua e permanente na qualidade de vida de crianças e adolescentes: revisão de literatura. Rev. bras. odontol. 72(1-2). 70-75. http://revodonto.bvsalud.org/scielo.php?script=sci_arttext\&pid=S0034-72722015000100014

Muller, R. A., Feres, M. A., Portella, M. Q. \& Feres, R. (2010). As má-oclusões e as desordens da articulação temporomandibular. Rev. Ortodontia. 43(4). 369-374. https://pesquisa.bvsalud.org/portal/resource/pt/lil-711938

Pereira, A. S., Shitsuka, D. M., Parreira, F. J. \& Shitsuka, R. (2018). Metodologia da pesquisa científica UFSM. https://repositorio.ufsm.br/bitstream/handle/1/ 15824/Lic_Computacao_Metodologia-Pesquisa-Cientifica.pdf?sequence=1

Peres, K. G., Frazão, P. \& Roncalli, A. G. (2013). Padrão epidemiológico das oclusopatias muito graves em adolescentes brasileiros. Revista de Saúde Pública. 47(3). 109-117. https://doi.org/10.1590/S0034-8910.2013047004366 
Rebouças, A. G., Zanin, L., Ambrosano, G. M, \& Flório, F. M. (2017). Fatores individuais associados à má oclusão em adolescentes. Ciênc. Saúde coletiva. 22(11). 3723-3732. https://doi.org/10.1590/1413-812320172211.04972016

Sharma, A., Mathur, A., Batra, M., Makkar, D. K., Aggarwal, V. P., Goyal, N. \& Kaur, P. (2017). Avaliação objetiva e subjetiva da necessidade de tratamento ortodôntico do adolescente e seu impacto sobre a autoestima. Rev. paul. pediatr. 35(1). 86-91. https://doi.org/10.1590/1984-0462/;2017;35;1;00003

Silva, A. L. Ó \& Padilha, W. W. N. (2018). Abordagens da produção científica odontológica brasileira sobre os efeitos da má oclusão e seus tratamentos na qualidade de vida. Rev. bras. ciênc. saúde. 22(4). 299-306. https://doi.org/10.22478/ufpb.2317-6032.2018v22n4.36231

Silveira, M. F., Freire, R. S., Nepomuceno, M. O., Martins, A. M. E., Marcopito, L. F. (2016). Gravidade da maloclusão em adolescentes: estudo de base populacional no norte de Minas Gerais, Brasil. Rev. Saúde Pública. 50(11). 1-10. https://doi.org/10.1590/S1518-8787.2016050005861

Sousa, J. P. \& Sousa, S. A. (2013). Prevalência de má oclusão em escolares de 7 a 9 anos de idade do Polo 1 da Rede Municipal de Ensino em João PessoaPB. Rev. Odontol. UNESP. 42(2). 117-123 https://pesquisa.bvsalud.org/portal/resource/pt/lil-674674

Spezzia, S., Porto, L. C., Oliveira, P. O. \& Weiler, R. M. (2014). Disfunção Temporomandibular na Adolescência. Portal Metodista: Odonto. 22(43-44). 5361. https://doi.org/10.15603/2176-1000/odonto.v22n43-44p53-61

Tristão, S. K., Magno, M. B., Pintor, A. V. B., Christovam, I. F. O., Ferreira, D. M. T. P., Maia, L. C. \& Souza, I. P. R. (2020). Is there a relationship between malocclusion and bullying? A systematic review. Progress in orthodontics. 21:26. 1-13. https://doi.org/10.1186/s40510-020-00323-7

Trize, D. M., Calabria, M. P., Franzolin, S. O. B., Cunha, C. O. \& Marta, S. N. (2018). A disfunção temporomandibular afeta a qualidade de vida? Einstein (São Paulo). 16(4). 1-6. https://doi.org/10.31744/einstein_journal/2018AO4339

Viana, M. O., Lima, E. I., Menezes, J. N. R. \& Olegario, N. B. C. (2015). Avaliação de sinais e sintomas da disfunção temporomandibular e sua relação com a postura cervical. Rev. odontol. UNESP. 44(3). 125-130. https://doi.org/10.1590/1807-2577.1071

Zou, J., Meng, M., Law, C. S., Rao, Y. \& Zhou, X. (2018) Common dental diseases in children and malocclusion. Int. J. Oral Sci. 10(1). 1-7. http://doi.org/10.1038/s41368-018-0012-3 